\title{
Cardiac Papillary Fibroelastoma with Coronary Artery Anomaly: A Case Report
}

\author{
Hongfei Xu, ${ }^{1 *}$ Liangwei Chen, ${ }^{1 *}$ Chengmeng Ye, ${ }^{1}$ Kwabena Gyabaah Owusu-Ansah, ${ }^{2}$ Yiming Ni, ${ }^{1}$ \\ Weidong $\mathrm{Li}^{1}$
}

Departments of ${ }^{1}$ Thoracic and Cardiovascular Surgery and ${ }^{2}$ General Surgery, First Affiliated Hospital of Zhejiang University, Hangzhou, China

\section{ABSTRACT}

Primary cardiac tumors are extremely rare with occurrence range of about $0.0017-0.28 \%$. Papillary fibroelastoma is a benign cardiac neoplasms. Improvement in the early diagnosis has been made possible with the help of high-resolution imaging technology, such as transesophageal echocardiography, combined with ischemic or embolic complications which patients typically present with clinically. We herein present a 51-year-old female patient with a papillary fibroelastoma on the aortic cusp, with its origin from the left coronary sinus. Her only clinical manifestation was angina-like chest pain and syncope. Surgical resection with aortic valve replacement due to the defect as a result of the resected tumor was performed with the patient on cardiopulmonary bypass. The patient recovered uneventfully and was discharged. A 2-year followup showed an intact valve without tumor recurrence.

\section{INTRODUCTION}

Primary cardiac tumors are considered extremely rare with a prevalence of about $0.0017-0.28 \%$. Papillary fibroelastomas (PFE) are considered to be the second most common cardiac valvular benign neoplasm after myxomas [Grinda 1999; Shi 2016]. More PFEs have recently been diagnosed with higherresolution imaging [Sun 2001]. We report a 51-year-old female patient with $\mathrm{PFE}$ on the aortic valve, originating from the left coronary sinus, which caused recurrent episodes of angina-like chest pain and which was successfully dealt with, resecting the mass on cardiopulmonary bypass.

\section{CASE REPORT}

A 51-year-old Chinese female with no family history of cardiovascular disease developed atypical chest pain, chest tightness, and dyspnea after activity 5 years prior. She

Received Fuly 5, 2016; received in revised form February 25, 2017; accepted March 3, 2017.

*Hongfei $X u$ and Liangwei Chen contributed equally to this work.

Correspondence: Weidong Li, Department of Thoracic and Cardiovascular Surgery, First Affiliated Hospital of Zhejiang University, Qing Chun Road 79, Hangzhou, China 310003; 86-137-35804465; fax: 86-571-28918695 (e-mail address: 11318287@zju.edu.cn). experienced weakness with cold sweat during the episode. At a local hospital, she was diagnosed with myocardial infarction based on findings on her electrocardiograph (ECG). Her symptoms were relieved by rosuvastatin, metoprolol, and nitroglycerin. The patient experienced a second episode two years later with discomfort after activity and she lost consciousness for about 5 minutes. Afterward she experienced weekly episodes of chest tightness, dyspnea, and hand numbness. Her symptoms continued without remission even after prescription medication for about two months prior to her presentation to our hospital. At our center, cardiac ultrasound showed a well-demarcated, homogeneous hyperechoic lesion on the aortic valve. 3D ultrasonography and transesophageal echocardiography (TEE) were performed which confirmed a mass with a pedicle attached to the aortic valve root close to the left coronary sinus wall. Primary diagnosis of an endocardial fibroelastosis fibroma was first considered (Figure, A). Computed tomographic angiography (CTA) showed the right coronary artery originating from the left coronary sinus without any signs of coronary atherosclerotic heart disease (CAHD) (Figure, B-C).

Surgical resection was performed via a median sternotomy and standard cardiopulmonary bypass. After opening the pericardium, the RCA and the LCA were both found originating from the left coronary sinus, $1 \mathrm{~cm}$ apart, with no obvious evidence of stenosis or calcium plaque. A mass about $1 \mathrm{~cm}$ in diameter with tiny tentacle-like fronds attached tightly to the edge of the left aortic valve was found (Figure, D). The tumor was firmly attached to the valve tissue through this short wide pedicle, which made it difficult to shave off completely while preserving the leaflet integrally. Therefore, for the purpose of minimizing the recurrent chance of the tumor, a decision was made to resect the tumor along with part of the leaflet tissue. Due to the lesion caused by the resection, an aortic valvularplasty procedure couldn't be implement. Thus, a decision to replace the aortic valve was made and the valve was replaced by a $19 \mathrm{~mm}$ St. Jude Regent mechanical valve prosthesis. The excised tumor was friable with a yellowish and jellylike appearance (Figure, D). The resected tumor had a characteristic frond-like appearance and resembled a sea anemone with a villous surface when placed in saline (Figure, E). After histopathologic examination, a definitive diagnosis of papillary fibroelastoma was made (Figure, F). After surgery, the patient recovered uneventfully and was discharged. Her 2-year follow-up exam was unremarkable and without evidence of tumor recurrence. 

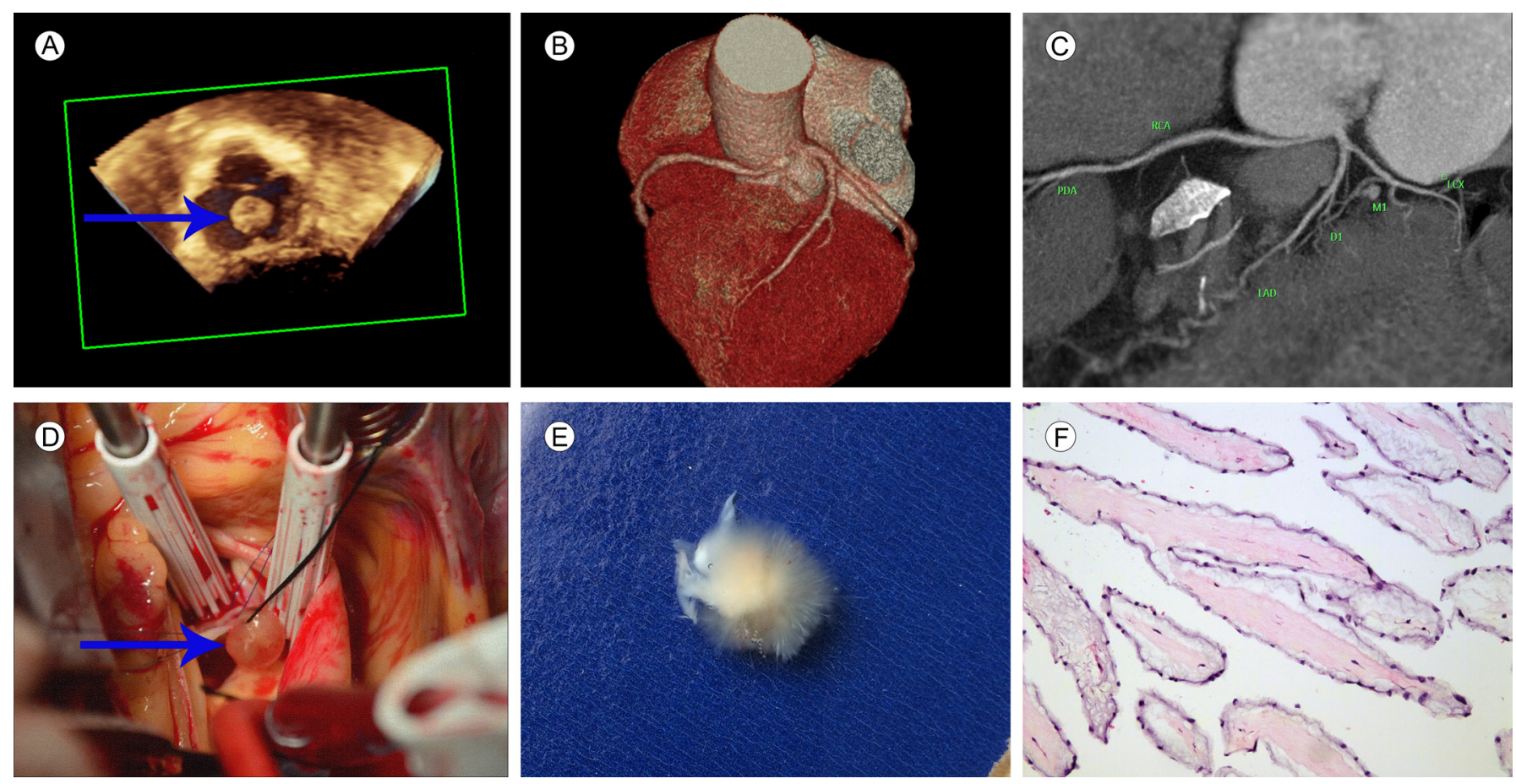

(A) Transesophageal echocardiography (TEE) showing a nodular mobile mass (blue arrow) at the aortic root, near the left coronary sinus wall. (B-C) Computed tomography angiography (CTA) depicting the right coronary artery originating from the left coronary sinus, $1 \mathrm{~cm}$ apart from the opening of the left coronary artery. There were no signs of obvious stenosis or calcium plaque in either coronary arteries. (D) Yellowish jelly-like mass on the aortic valve seen intraoperatively (blue arrow). (E) The anemone-like appearance with villi on its surface when submerged in water. (F) Histological section showing a single layer of endocardial cells covering the surface of cavities filled with mature collagen.

\section{DISCUSSION}

Valvular tumors were first described by Yater in 1931 [Yater 1931]. Cardiac papillary fibroelastomas are benign endocardial papilloma predominantly affecting the cardiac valves with the aortic valve [Gowda 2003]. They may arise from various tissue components such as elastic fibers, fibrous tissue, or smooth muscle cells in ventricular outflow tract, the ostium of right and left coronary artery. Characteristically, PFE resembles a sea anemone with multiple papillary fronds and is usually attached to the valve or endocardium by a short pedicle or stalk [Grinda 1999]. However, the origin of PFEs remains unclear with variable inferences mentioned in the literature. They have been regarded as organized thrombi or masses resulting from unusual endocardial response to hemodynamic trauma or infection [Bicer 2009]. Nevertheless, some authors consider PFEs as true neoplasms.

PFEs are usually asymptomatic, but can sometimes cause ischemic or thromboembolic symptoms, such as transient ischemic attack, stroke, angina, myocardial infarction, heart failure, presyncope, syncope, and blindness [Hirotsugu 2011]. Life-threatening complications such as cardiac arrest and sudden death have also been reported.

In our patient, preoperatively CTA showed no signs of coronary stenosis or calcium plaque. Also, while perfusing antegrade cold blood cardioplegia during the surgery, the perfusing pressure, resistance, and flux were all within normal range. In our opinion, the patient's symptoms, including chest pain, chest tightness, and dyspnea, were not caused by the coronary anomaly. These symptoms were mainly due to the tumor obstructing or limiting blood flow through the coronary artery orifice, resulting in partial myocardial ischemia. Another theory might be that the tentacles of the mass could be carried off with the current as blood flew across the mass and then passed into the coronary artery, resulting in a relatively narrow orifice of the coronary artery, eventually leading to partial myocardial ischemia. Therefore, we did not consider intervention on the coronary anomaly. The patient was weaned from the bypass successfully, confirming our ideas.

PFEs can be diagnosed with 2- or 3-dimensional echocardiography and TEE. MRI and CTA have recently been included as new imaging modalities to improve the diagnostic accuracy of PFEs [Chia 2009].

Although considered benign, the effect of the tumor and its location in the heart can influence blood flow, causing a series of ischemic symptoms like chest pain and syncope. Although a debate over the necessity for surgical treatment of asymptomatic patients still exists, there is no doubt that the presence of a papillary fibroelastoma is an absolute indication for resection, regardless of whether it is suspected of having caused symptoms or not. Therefore, surgical resection is curative and recommended in symptomatic patients to prevent further ischemic or thromboembolic events [Wang 2016]. Preventing fragmentation of mass during surgery can be key in reducing the postoperative complications. In addition, damage of the valve during excision may need repair or 
replacement [Boodhwani 2007]. In our case, the tumor was difficult to shave off and we had to perform a resection of the tumor. The left coronary cusp was significantly damaged after resection, and hence the aortic valve was replaced in anticipation of preventing aortic regurgitation.

We speculate that the angina-like chest pain or the syncope was induced by transient occlusion of the left coronary orifice by the tumor, which if left untreated can cause sudden cardiac death. At 2-year follow-up, our patient remained asymptomatic and her recent TTE showed no tumor recurrence.

\section{Conclusion}

Cardiac PFEs are rare and usually asymptomatic. However, they can cause ischemic or thromboembolic symptoms in some patients. Diagnosis should be combined with several imaging modalities to help differentiate it from other cardiac tumors. Our patient presented with angina-like chest pain and syncope. It is our belief that resection of the tumor is necessary not only in symptomatic patients but also in asymptomatic patients to avoid vascular or embolic complications.

\section{ACKNOWLEDGEMENT}

The authors thank Chengcheng Li (Zhejiang University) for English editing.

\section{REFERENCES}

Bicer M, Cikirikcioglu M, Pektok E, Muller H, Dettwiler S, Kalangos A. 2009. Papillary fibroelastoma of the left atrial wall: a case report. J
Cardiothorac Surg 4:28.

Boodhwani M, Veinot JP, Hendry PJ. 2007. Surgical approach to cardiac papillary fibroelsatomas. Can J Cardiol 23:301-2.

Chia PL. 2009. Incidental finding of an aortic valve mass on 64-slice computed tomographic coronary angiography. Annals Academy Med 38:926-7.

Gowda RM1, Khan IA, Nair CK, Mehta NJ, Vasavada BC, Sacchi TJ. 2003. Cardiac papillary fibroelastoma: A comprehensive analysis of 725 cases. Am Heart J 146:404-10.

Grinda JM, Couetil JP, Chauvaud S, et al. 1999. Cardiac valve papillary fibroelastoma: surgical excision for revealed or potential embolization. J Thorac Cardiovasc Surg 117:106-10.

Hirotsugu K, Tamotsu K, Homare Y, et al. 2011. Successful early resection of cardiac papillary fibroelastoma. Gen Thorac Cardiovasc Surg 59:191-4.

Shi J, Bai ZX, Zhang BG, Guo YQ. 2016. Papillary fibroelastoma of the aortic valve in association with rheumatic heart disease: a case report. J Cardiothorac Surg 11:6.

Sun JP, Asher CR, Yang XS, Cheng GG, Scalia GM, Massed AG. 2001. Clinical and echocardiographic characteristics of papillary fibroelastomas: a retrospective and prospective study in 162 patients. Circulation 103:2687-93.

Wang Y, Wang XF, Xiao YB. 2016. Surgical treatment of primary cardiac valve tumor: early and late results in eight patients. J Cardiothorac Surg $11: 31$.

Yater WM. 1931. Tumors of the heart and pericardium: pathology, symptomatology and report of nine cases. Arch Intern Med 48:627-66. 